\title{
The Human Rights Initiative: A Medical Student Clinic Documenting Sequelae of Torture for Asylum Seekers
}

Greg Raczkowski, MS; Maria Coluccio, MA; Kim Griswold, MD, MPH; Representing

the Jacobs School of Medicine and Biomedical Sciences of the University at Buffalo

\section{Background}

The ongoing refugee and migrant crisis has led to the displacement of millions worldwide, with estimates that, of the 70.8 million individuals displaced, 3.5 million are seeking asylum ${ }^{1}$. Many of these asylum seekers have experienced physical and/or psychological torture often state-sponsored - in their home countries. The experience of suffering through torture is often sufficient to qualify for asylum. However, it is often difficult for the asylee to prove in court that he or she has actually experienced this trauma. Additionally, the symptomatology of psychiatric disorders caused by torture can involve memory loss or an inability to accurately describe the traumatic event - a feature that may affect a judge's decision in granting asylum. Evaluation and documentation of the physical and psychological sequelae by a medical professional provides objective assessment of an asylum seeker's symptoms and often lends credence to his or her claims. These evaluations have been shown to significantly increase the likelihood that an individual is granted asylum².

\section{Aims}

The Human Rights Initiative (HRI) at the University at Buffalo was founded in 2014 and is the $4^{\text {th }}$ most active Physicians for Human Rights-affiliated medical student run asylum clinic in the United States. $\mathrm{HRI}$ works with immigration attorneys and their clients to arrange physician-led forensic evaluations in order to provide an objective assessment and legal affidavit of the lasting physical and psychiatric sequelae of torture. These expert assessments are used as evidence of persecution in the asylum seeker's home country and directly affect their asylum decision.

\section{Objectives}

Due to the ongoing migrant crisis and the vulnerability of this population, it is important to have accurate information to drive both policy decisions and interventions to aid this group. To that end, the objective of this study was to provide descriptive analysis on the group of asylum seekers who received a forensic evaluation from HRI. The data generated includes demographics and prevalence of both exposures to physical trauma and psychiatric conditions.

\section{Materials \& Methods}

A retrospective analysis was performed on all forensic evaluations performed by HRI since 2014 and seven finalized legal affidavits were descriptively analyzed. Prevalence of symptoms meeting DSM-V criteria for a psychiatric disorder and of significant clinical sequelae related to Physical Violence, Emotional Violence, and Female Genital Mutilation (FGM) was noted. "Levels of consistency" were determined by trained clinicians in accordance with the Istanbul Protocol ${ }^{3}$.

\section{Results}

- HRI's approval rate of $84.6 \%$ is significantly higher than the 2017 U.S. average of $28 \% 4$

- Analyzing a subset, $43 \%$ met DSM-V criteria for PTSD, and many also suffered from comorbid MDD

- Refer to Table 1 and Figures 1.a - 1.c for summary of analysis

Table 1. Demographics of Asylum Seekers undergoing Forensic Evaluations with HRI

\begin{tabular}{l|c|l}
\multicolumn{2}{c}{ Forensic Evaluations with HR } \\
\hline Variable & $\mathbf{N}$ & Percentage \\
\hline Total Evaluations & $\mathbf{1 5 9}$ & \\
\hline Medical & 69 & $43.4 \%$ \\
\hline Psychiatric & 90 & $56.6 \%$ \\
\hline Gender & & \\
\hline Male & 67 & $60.4 \%$ \\
\hline Female & 44 & $39.6 \%$ \\
\hline Asylum Status & 26 & \\
\hline Granted & 22 & $84.6 \%$ \\
\hline Denied & 4 & $15.4 \%$
\end{tabular}

\section{Conclusions}

Based on our findings, psychiatric illness is extremely common amongst asylum seekers undergoing forensic evaluations. Circumstances leading to asylum are often traumatic, thus it is possible that this is generalizable to all asylum seekers. Further research must be done on this vulnerable population to investigate this point, as interventions designed at increasing access to mental health care may have a considerable impact. The significantly increased asylum acceptance rate and high prevalence of psychiatric illness also demonstrates the value that forensic evaluations offer asylum candidates; many diagnoses may have otherwise been overlooked and not been used for their asylum decision.

Figure 1. Prevalence of Clinical Sequelae Associated with Exposure to Violence

A. Physical Violence

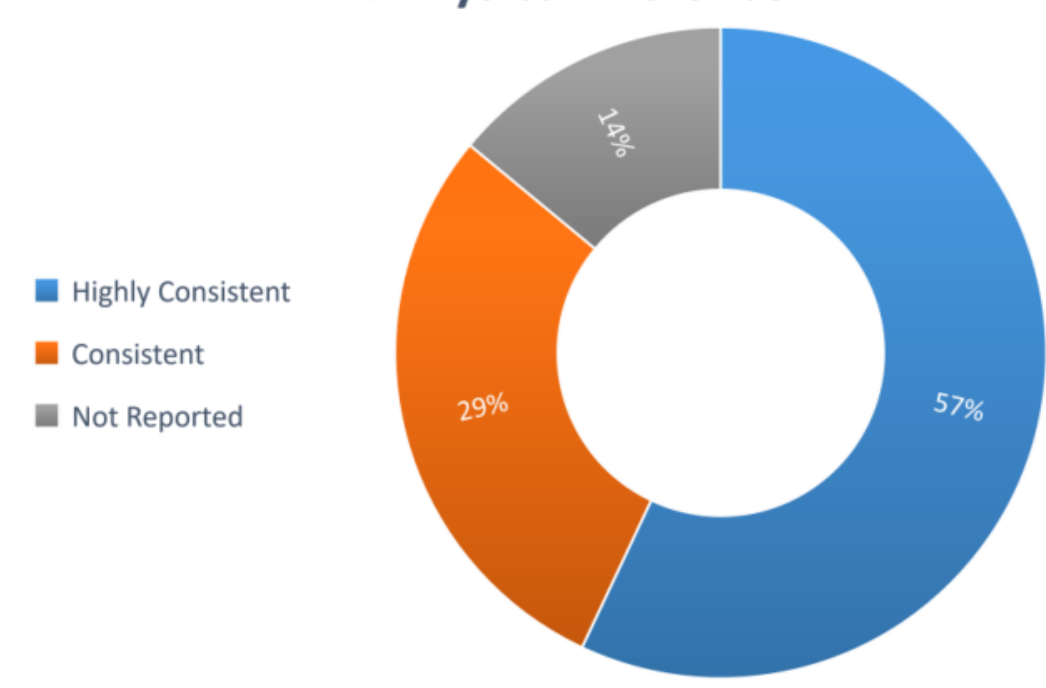

B. Emotional Violence

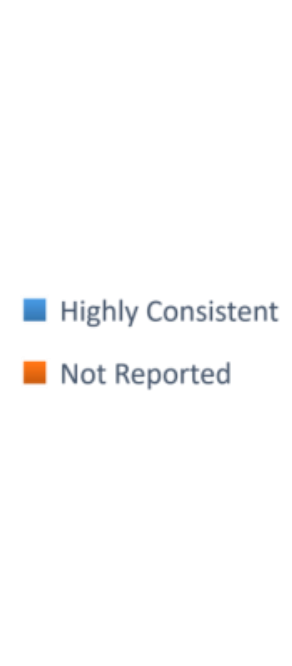

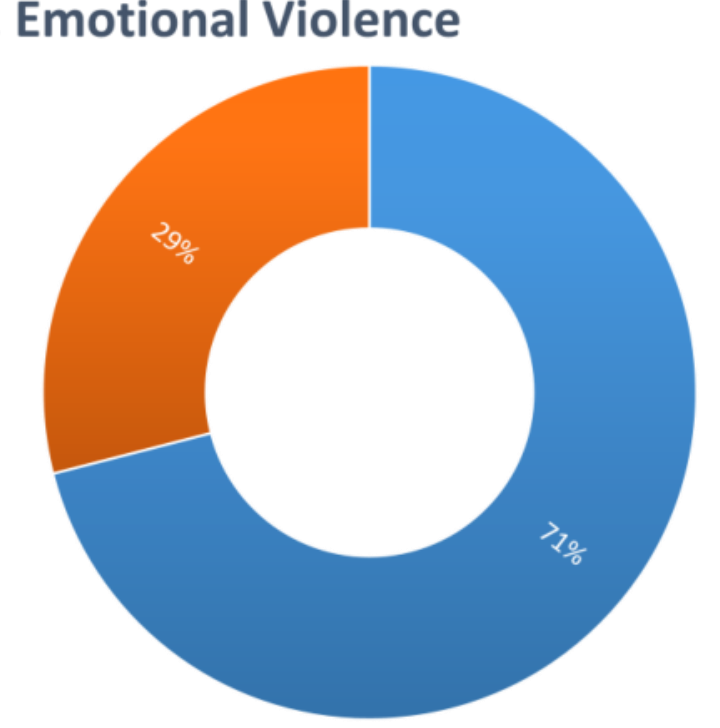

C. Female Genital Mutilation
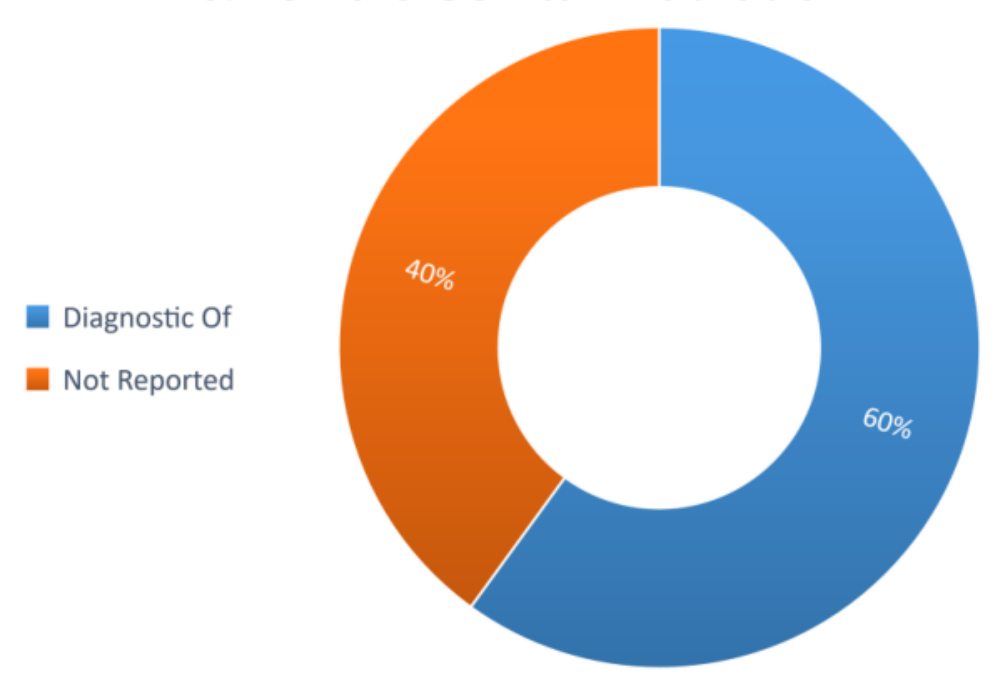

1. "UNHCR Statistical Yearbooks." United Nations High Commissioner for Refugees, www.unhcr.org/statistical-yearbooks.html.

2. Lustig SL, Kureshi S, Delucchi KL, lacopino V, Morse SC. Asylum grant rates following medical evaluations of maltreatment among political asylum applicants in the United States. J Immigr Minor Health. 2008;10(1):7-15.

3. UN Office of the High Commissioner for Human Rights (OHCHR), Manual on the Effective Investigation and Documentation of Torture and Other Cruel, Inhuman or Degrading Treatment or Punishment ("Istanbul Protocol"), 2004. 